\title{
GONIOTOMY FOR THE RELIEF OF CONGENITAL GLAUCOMA
}

BY

\author{
OtTO BARKan
}

SAN FRANCISCO

CongenITAL or infantile glaucoma (hydrophthalmos, buphthalmos), has been among the most hopeless of ocular conditions requiring surgery. In the past, rare cases in which sight was preserved by operation or by spontaneous arrest appear to be exceptions that prove the rule. Trephination has been unsuccessful and often disastrous, cyclodialysis ineffective. A few cases of normalisation of pressure over a period of years by iridencleisis have been reported.

In recent articles 1,2 observations on congenital glaucoma and the results obtained during the past ten years by goniotomy performed on 76 eyes presenting congenital glaucoma were published. In 66 of these pressure was normalised and vision maintained or restored. In ten the operation was unsuccessful.

Goniotomy is an operation for stripping or peeling embryonic tissue from the angle wall. If the cornea is clear the operation is performed under direct vision with the aid of a prismatic contact glass specially devised for this purpose. In this series the operation was performed under the contact glass on 20 eyes. On 56 eyes the operation was performed without the contact glass because of corneal cloudiness.*

It is the purpose of the present article to discuss the diagnosis and cause of reduced vision'in infantile glaucoma and to stress the urgent need of early adequate operation. The technique of goniotomy is described. Indications for the operation and its mode of action, which are essential to an understanding of its principle, are set forth.

The operation is exacting. It requires teamwork and demands meticulous attention to detail of technique in order to obtain a high incidence of good results and to avoid injury which may have tragic consequences. Experience with gonioscopy and acquaintance with the varying picture of congenital glaucoma are helpful.

\section{Diagnosis}

The early symptoms in congenital or infantile glaucoma are often of a congestive and irritative nature. In the present series out of 87 eyes, 70 showed congestive symptoms at the time of onset.

* The approach to the angle across the chamber was first introduced by de Vincentiis in 1892 and some successes were reported. The method later fell into disuse. 
Congestive symptoms caused by increased pressure consist of :

1. Corneal cloudiness, minor or extreme.

2. Hyperaemia of the bulbar conjunctiva, slight to moderate.

3. Photophobia, which may be extreme.

4. Epiphora.

5. Blepharospasm.

In 30 eyes cloudy cornea was present at birth. In 35 eyes the onset of congestive symptoms appeared between the first and fourth

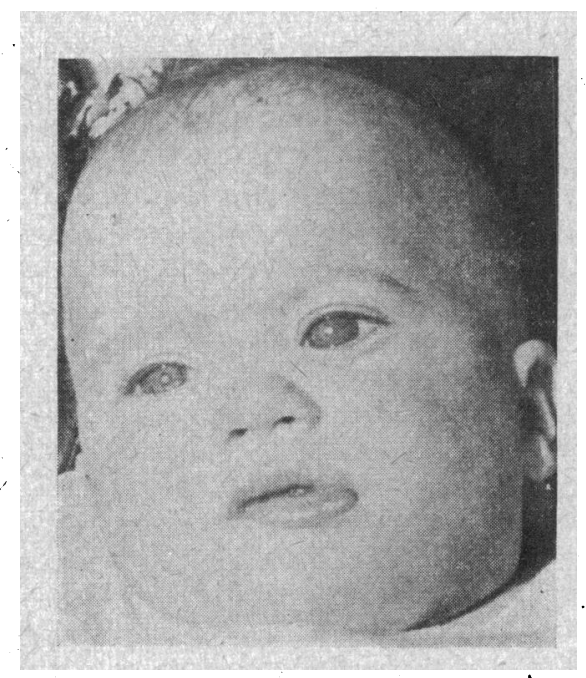

FIG. 1.

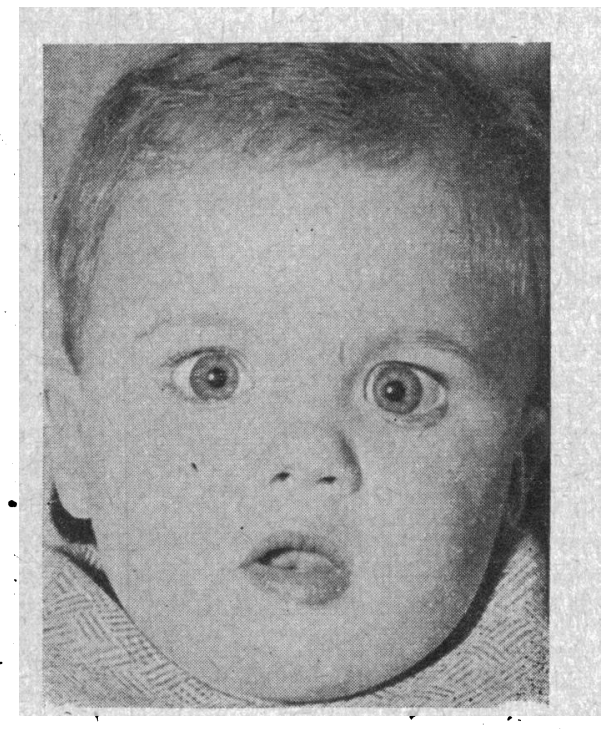

FIG. 2

(Pre-operative).-P.O'Q., aged 5 months, first seen July 25, 1944. Cloudy corneae developed six days before. Patient showed slight photophobia and tearing since birth. Pupils are in miosis due to three instillations of 5 per cent. solution of prostigmine every three hours.

(Post-operative).-P.O'Q., aged $2 \frac{1}{2}$ years. From August 9, 1944, to October 2, 1945, four goniotomies were performed on each eye : The last operation on the right eye was nine months ago, and on the left eye eighteen months ago. Note clear corneae, normal-appearing eyes and complete absence of symptoms. Tension (McLean) March 17, 1946, was $34 \mathrm{~mm}$. in the right eye and $34 \mathrm{~mm}$. in the left eye without use of miotics. Refraction owas -2.00 sphere in each eye. Ophthalmoscopic examination showed normal appearance of heads of optic nerves.

months. In 11 eyes the onset was between the fourth and ninth months. Some increase of pressure and enlargement of the eyeballs was present before congestive symptoms appeared, since these 
Goniotomy For the Relief of Congenital Glaucoma 703

children are often described as having had especially beautiful (i.e., large) eyes. This appearance in otherwise normal looking eyes should arouse suspicion. Usually, however, the onset of congestive symptoms is the feature which calls attention

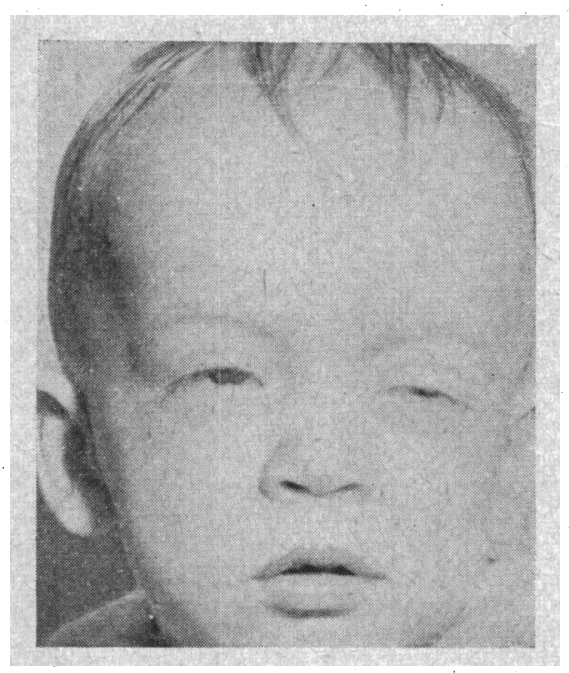

FIG. 3.

(Pre-operative).-T.C., aged 11 months, first seen July 10, 1942. Two months previously, on May 11, 1942, cornea of left eye became cloudy overnight. There were photophobia and tearing. The eye could be opened with difficulty; the bulbus was enlarged.

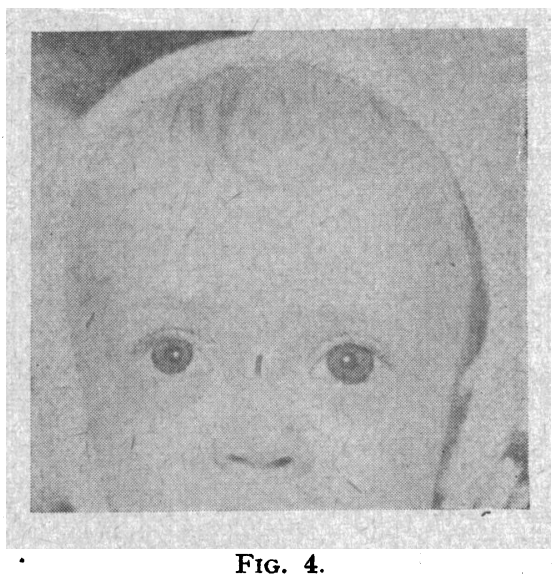

(Post-operative).--T.C., aged 14 months, three months after goniotomy was performed on the left eye, on August 21, 1942. Note clear cornea. The left eye is slightly enlarged, but is otherwise normal in appearance. On May 25, 1944, twenty-one months after the operation, tension (McLean) was 29 $\mathrm{mm}$. in the right eye and $28 \mathrm{~mm}$. in the left eye without use of miotics. Refraction was as follows : right eye, $-1.00 \mathrm{sph} .-1.00$ cyl. axis $180^{\circ}$; left eye -3.50 sphere. Gonioscopy of the left eye showed stripping of angle in one-third of its circumference. Ophthalmoscopic examination showed wide shallow excavation of the head of the optic nerve of the left eye but no atrophy.

to the condition; the onset is often rapid and may be even sudden. Such was the case in six infants in this series in whom cloudiness of the cornea was discovered by the mother when the infant was taken up in the morning; the eyes had been clear the previous night. In one case the cloudiness was discovered when the infant was taken up from its afternoon nap. 
The cloudiness of the cornea is often associated with photophobia, which may be so intense that the eyes cannot be opened except in the dark. For this reason it was impossible to obtain pre-operative photographs in many cases. Fig. 1 illustrates moderate cloudiness of the cornea. Fig. 2 shows the same patient

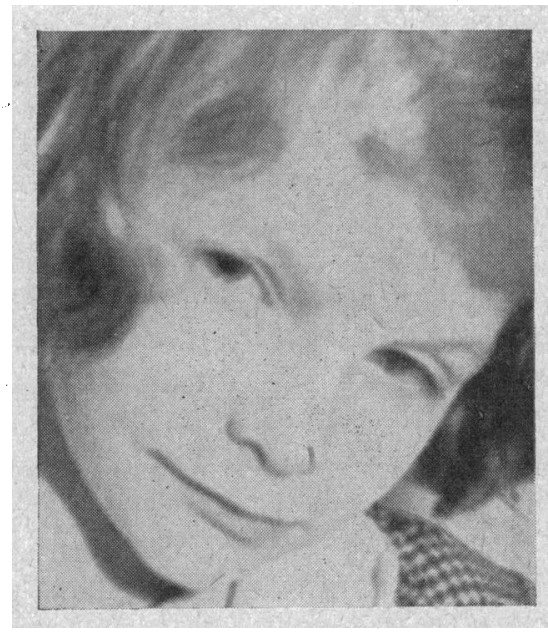

FIG. 5.

(Pre-operative).-J.V., aged 6 years. Since birth left eye was slightly larger and cloudy; there was some tearing. When first seen May 10, 1943, tension in right eye was normal, and in left eye was -1 to $i-2$. Photograph shows patient avoiding light. There is some tearing.

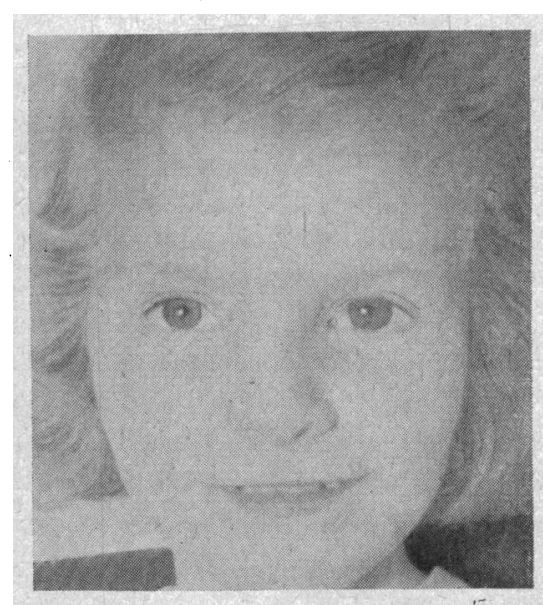

FIG. 6.

(Post-operative).-J.V., aged 7 years. Goniotomy was performed on left eye May 12, 1943. Photograph shows patient one year after operation. Note clear cornea, absence of photophobia and normal appearance. Patient was last seen July 2, 1946, three years, two months after operation; excellent condition had been maintained; tension in right eye was 31 , in left eye $\mathbf{3 3}$ (McLean), without miotics; media were clear; ophthalmoscopic examination showed wide shallow excavation of head of optic nerve not extending to the rim; colour of disc was good. Vision without correction, in right eye, is 0.8 ; in left eye, 0.4. With correction, vision in left eye is $-0.50 \mathrm{sph}$. -0.75 cyl. axis $90^{\circ},=0.6$ and $\mathrm{J} .-2$.

post-operatively. Figs. 3 and 5 illustrate pre-operative congestive signs of minor degree which permitted of photography. Figs. 4 and 6 show the same patients post-operatively. In the case of T.M., aged 11 months, the eyes were of normal appearance until the age of 6 months when, following whooping cough and pneumonia, the mother noticed cloudy corneae. From then on the child was 
extremely photophobic. It spent its life playing at night in a dark room and sleeping during the day. When brought for examination it kept its head buried in its mother's bosom. A glimpse of the eyes could be obtained only by separation of the lids by force because of the intense blepharospasm. Ten days after goniotomy the corneae were clear and photophobia almost absent. Fig. 7

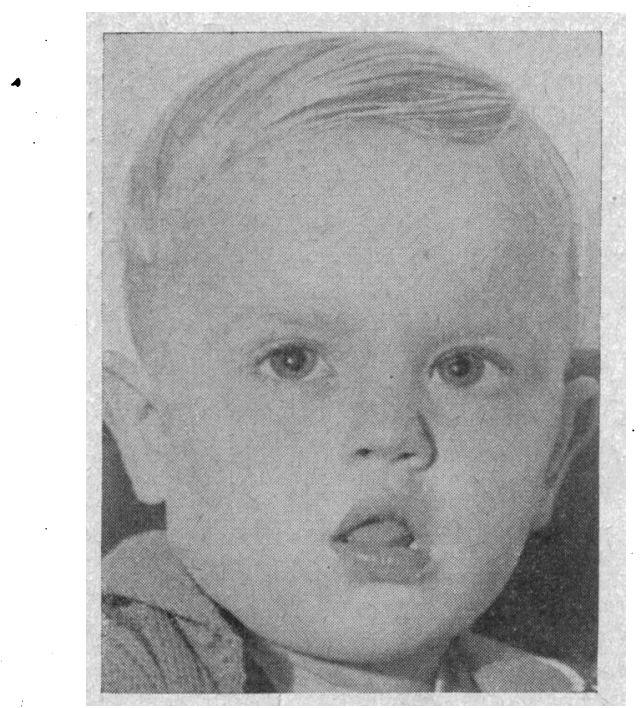

FIG. 7.

(Post-operative).-T.M., aged 11 months, first seen July 14, 1943. After whooping cough and pneumonia at the age of 6 months both corneae became cloudy and photophobia was extreme, so that the eyes could not be opened in daylight. Goniotomy was performed six months after the onset of symptoms, on the right eye on May 17 and on the left eye on May 20,1943. Nine days after the operation the corneae were clear, eyes were open and patient tolerated light well. Eyes were normal in appearance, except for slightly increased size. There was no sign of corneal opacity, but retinoscopy showed slight irregular corneal astigmatism. There was myopia of approximately -2.00 sphere. On September 27, 1944 (sixteen months after the operation), tension (McLean) was $29 \mathrm{~mm}$. in the right eye and $20 \mathrm{~mm}$. in the left eye without miotics. In June, 1946 (three years after the operation) a letter from Texas reported patient's condition to be excellent to date.

shows this patient 16 months post-operatively. The corneae are clear; vision is excellent.

The corneal cloudiness and lack of lustre which is characteristic of the early stage was shown by staining with fluorescein to be due chiefly to a disturbance of the epithelium. It appears that it is largely this disturbance, consisting of roughening of the corneal 
epithelium, which is the cause of the irritative symptoms and photophobia.

A physician who first sees the child with these congestive symptoms is apt to make a diagnosis of conjunctivitis, blepharitis or keratitis. He is not prone to think of glaucoma because the term " buphthalmos" is associated in his mind only with enlargement of the eyeball. The resulting delay in arriving at the correct diagnosis and instituting proper and adequate treatment may be tragic, for by the time the eyeball is strikingly enlarged the patient is usually well on his way to semi or total blindness: Therefore; in every case in infants in which irritative phenomena or corneal cloudiness are present, even though the bulbus does not appear enlarged, glaucoma must be suspected and intra-ocular pressure tested with a tonometer.

Tension should always be measured under ether anaesthesia which must be sufficient to assure complete relaxation at the moment of measurement. Other general anaesthetic agents have proved unsatisfactory. It has been found that tension may vary 10 to $15 \mathrm{~mm}$. of mercury, depending on the depth of anaesthesia, and only when the patient was completely relaxed was the tension constant. Cases have been observed in which increased tension due to insufficient anaesthesia led to an incorrect diagnosis of congenital glaucoma. In the present series of cases in which the pressure was elevated, prostigmine 5 per cent. was instilled in the eyes every three hours and goniotomy performed as soon as possible.

In cases in which the cornea is sufficiently transparent one finds on careful gonioscopic examination characteristic evidence of congenital glaucoma in the chamber angle.

\section{Cause of Reduced Vision in Infantile -Glaucoma}

Anderson ${ }^{3}$ has pointed out that the cause of reduced vision in infantile glaucoma is usually regarded as being optic atrophy and one or more disorders in addition. In the present series atrophy of the optic nerve and others of these disorders were not the original cause, but were the result of progressive enlargement of the eyeballs and of tissue degeneration associated with the late, advanced stage of the disease. The primary cause of reduced vision in most cases in the series was a disturbance of the cornea. This disturbance begins with corneal cloudiness (oedema?). It appears to coincide with the onset of increased intra-ocular pressure. Allowed to persist it is followed by permanent scarring of the cornea with associated irregular astigmatism and ensuing amblyopia of greater or less degree. In those cases in which corrieal cloudiness was relieved within a few days after onset acute vision developed. 


\section{EFFECTS OF HyPERTENSION}

(a) Corneal cloudiness is the main characteristic of the congestive phase of the early stage and at this stage is completely reversible surgically and occasionally by administration of miotics. The cloudiness usually occupies the central area. In severe cases it may cover the entire cornea, leaving only a strip adjoining the limbus clear. Fluorescein stains such corneae. Exposure to air, 'such as occurs when the blinking reflex is prevented while the patient is under general anaesthesia, increases the cloudiness and staining within a few seconds; if the lids are kept closed for a few minutes these decrease. That the corneal lesion is at least partially dependent on exposure is confirmed by its characteristic localization in the palpebral fissure zone. Abrasion of the epithelium in these cases shows a clear parenchyma in the denuded area. Another form of cloudiness appears in some cases to be due to an optical derangement of the corneal fibres. This cloudiness is evanescent. It can be produced by pressing on the bulbus; on relieving the pressure the cloudiness disappears.

The roughened epithelium appears to be the cause of the irritative symptoms which in turn are aggravated by exposure to air and by light. These irritative symptoms promptly disappeared following normalization of pressure, coincident with the-simultaneous restoration of corneal lustre and epithelial integrity. Clinically the child's comfort closely paralleled the epithelial integrity. Patients presenting high intra-ocular pressure and good corneal lustre are usually comfortable and show no trace of photophobia or other irritative signs. Patients presenting lower pressure and poor corneal lustre display irritative symptoms. The tolerance of the cornea to increased pressure manifests great individual variation. In some cases in which the tension is 50 McLean the cornea may remain clear and irritative, symptoms be absent during the course of many months; in others the development of the same degree of tension may immediately produce marked cloudiness and irritability.

Cloudiness of the corneae at this early age sometimes is associated with nystagmoid movements. In two cases these were relieved by clearing of the cornea. To illustrate : J.McL., born with cloudy corneae, presented marked nystagmoid movements when first seen at the age of five months. Three weeks after normalization of pressure in both eyes by goniotomy, these had greatly decreased. They disappeared almost entirely in the course of the next several months. In the case of J.H., aged three months when first examined, corneal cloudiness had developed two weeks previously. During the interval of 15 days which elapsed between 
operation on the two eyes nystagmoid movements made their appearance. Two weeks after operation on the second eye when pressure was normalized and the corneae were clear, these disappeared.

Cloudiness of the cornea requires quick relief (1) because of the rapidity with which it interferes with the development of vision, and (2) because the longer it exists the denser and more extensive will be the ensuing permanent scar. The present series shows that corneal cloudiness can be relieved and that in most cases formation of a permanent corneal opacity and development of amblyopia can be prevented in great measure by early diagnosis and prompt operation.

(b) The cloudiness becomes a permanent scar. This "late" milky opacification develops from the original reversible congestive cloudiness of the cornea when the latter has lasted sufficiently long to cause permanent damage to the corneal parenchyma. The prolonged distention per. se may also be a factor in its formation, or both factors may be operating concurrently. This type is reversible in part only. It involves chiefly the anterior layers of the corneal parenchyma. The epithelium shows normal to fair lustre and does not stain to fluorescein if the eye is in a non-congestive phase. Certain of these cases show relative absence of congestive symptoms because compensation has taken place. Pressure may actually be less than in the early stage, perhaps partially due to thinning of the tunics of the distended eye and possibly also to increased permeability of the occluding persistent meshwork in the angle resulting from stretching. The latter may be the mechanism, as has been suggested, which accounts for some of the rare cases of spontaneous arrest.

(c) Amblyopia. It is essential for the physician to keep in mind that a cloudy cornea during the first year of life, unless promptly relieved, retards the proper development of central vision at a most critical time. These cases illustrate the susceptibility of the development of visual function to normal entry of light through the refractive media in the first year of life. They stress the urgency of prompt and adequate surgical interference. They also show the need for diligent continued observation after pressure has been normalized, in order to detect beginning strabismus which could rapidly induce amblyopia unless proper treatment with complete occlusion of the fellow eye is instituted.

(d) Glaucomatous atrophy of the optic nerve was the exception in this series. It occurred only in those few cases in which normalization of pressure was unduly delayed. In the case of B.O'S. (Fig. 9), patient did not come to operation until the age of $4 \frac{1}{2}$ years. The left eye had been lost due to atrophy following a 


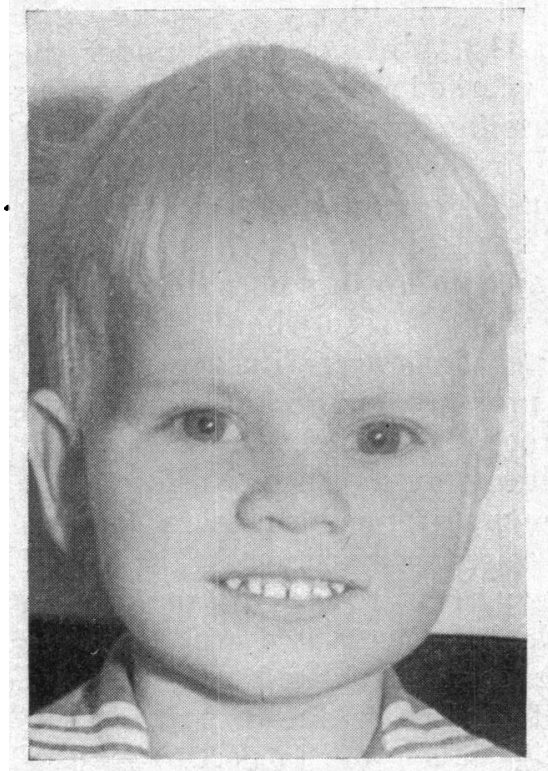

FIG. 8.

(Post-operative).-H.B., aged 7 months, when first seen on June 28, 1939. Three months previously the cornea of the left eye had become cloudy. Tension in the right eye was $20 \mathrm{~mm}$. and in the left $90 \mathrm{~mm}$. (McLean). Goniotomy was performed on the left eye on June 29, 1939. Drops of 5 per cent. solution of prostigmine were instilled every three hours in both eyes. On August 7, 1939, the tension (McLean) during ether anaesthesia was $50 \mathrm{~mm}$. in the right eye and $31 \mathrm{~mm}$. in the left. The cornea of the right eye was cloudy. Goniotomy was performed on the right eye. Three days after the operation the cornea of the right eye was clear. On November 27, 1939, three months after the operation the tension (McLean) in the right eye was $22 \mathrm{~mm}$. and in the left was $22 \mathrm{~mm}$. without miotics. On August 12, 1942, when the patient was aged 3 years 9 months, vision of the right eye without correction was 0.5 and of the left was $1 / 200$. When the patient was last seen, on June 12, 1946, when he was aged 7 years and 7 months, his condition was the same, and the tension was normal. The right eye was emmetropic, the left eye myopic $-\mathbf{3 . 5 0}$ sphere. The reduced vision of the left eye appeared to be due to amblyopia which developed during a transitory period of strabismus. Binocular fixation was present.

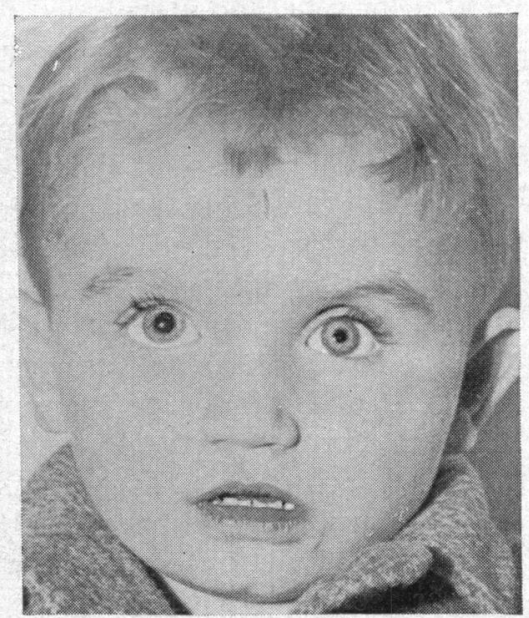

FIG. 9.

(Post-operative).-B.O'S., aged 4 years, first seen July 20, 1945. The left eye had been lost through trephine six months before. The cornea of the right eye was clear ; tension (McLean) with miotics was $70 \mathrm{~mm}$. Ophthalmoscopic examination showed glaucomatous excavation of the nerve head to the rim with slight pallor temporally. Vision was excellent, and visual fields were grossly normal. Goniotomy on the right eye with the contact glass was performed on October 12, 1945. Three months afterward tension (McLean) in the right eye was $26 \mathrm{~mm}$. without miotics. 
trephine operation performed at the age of four years. The right eye carried a pressure of $50 \mathrm{~mm}$. Hg, McLean while under the influence of miotics. It showed marked glaucomatous cupping of the nerve-head to the rim. Because the cornea was clear excellent central vision had developed. This has been maintained since normalization of pressure by means of goniotomy performed one year ago. It is interesting to note, incidentally, that the excavation of the nerve-head almost disappeared following normalization of pressure. In the case of B.P., who was first seen when 5 weeks old, repeated goniotomy and other operations were unsuccessful on one eye for two years; by the time pressure had became normalized optic atrophy had occurred. In the case of L.L., aged 3 years, bilateral trephination with total iridectomy had been performed at the age of 8 months without permanently reducing tension. Cyclodiathermy also failed permanently to reduce tension. At the age of 3 years goniotomy was performed; pressure was normalized in both eyes, congestive symptoms which had persisted until this time were completely relieved and the corneae cleared considerably. Moderate reduction of vision was found to be due in part to faint corneal scarring with irregular astigmatism and in part to glaucomatous atrophy which had occurred as a result of the protracted period of time during which the pressure remained elevated.

Ruptures or tears of Descemet's membrane, which are prominently referred to in the literature describing the pathological anatomy of " buphthalmos," were absent in this series of infantile glaucoma. It appears that tears of Descemet's membrane in infantile glaucoma do not occur before marked distension and degenerative changes have taken place. These conditions did not exist at the time goniotomy was performed in this series, which probably accounts for the absence of tears. If this observation is correct, rupture of Descemet's membrane plays no rôle in diagnosis at the time when diagnosis is of therapeutic importance.

\section{Mode of Action of Goniotomy}

In congenital glaucoma the angle presents a characteristic gonioscopic appearance which shows little individual variation. Other anomalies such as gross anterior adhesions were present in only 2 out of 77 eyes examined by the writer. The characteristic condition appears to consist of an abnormal insertion of the iris, by means of adventitious mesoblastic tissue into the angle wall anterior to its place of normal insertion.* Gonioscopic examination

* Gonioscopy of normal eyes of infants indicates that the chamber angle does not assume adult appearance until after the age of several months indicating that the uveal meshwork continues to regress after birth: This appearance can in most cases be distinguished from the pathological persistence of uveal tissue which is present in congenital glaucoma. 
with hand slit-lamp and binocular microscope held in the hand reveals that the iris continues in a horizontal plane until it reaches the posterior surface of the cornea. This is in contrast to dipping backwards of the iris as seen in the adult, thus forming a sinus or the so-called chamber angle. The point of attachment corresponds to the position of the anterior border ring of Schwalbe. The area of transition from the anterior surface of the iris to the posterior surface of the cornea consists of a gelatinous looking semi-transparent substance. This area may be so narrow that it barely constitutes a line. It may be wider, in which case the beam of light shows the tissue to be in a plane anterior or axial to the true angle

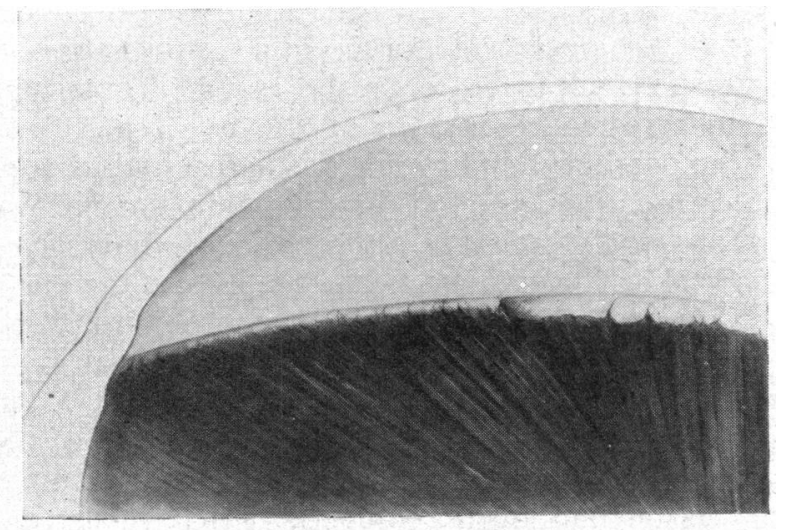

FIG. 10.

Drawing shows post-operative microgonioscopic appearance at the point of transition between the area on the right in which the angle has been stripped and the neighbouring untouched region.

wall the presence of which can in some cases be discerned in a deeper plane. Delicate arborescences of uveal meshwork which cross the angle can be seen resting on the semi-transparent substance which fills the angle behind them. From this finding and from the flare of the beam of the slit-lamp traversing it, it is evident that the angle is not an optically empty space. This fact becomes especially evident post-operatively at the point of transition between the area in which the angle has been stripped and the neighbouring untouched region (Fig. 10). The gonioscopic appearance corresponds to that which would be expected from a study of histological sections of eyes affected with congenital glaucoma enucleated in the early stages. Post-operative gonioscopy in the successful cases revealed that the procedure had been effective in stripping the persistent embryonic tissue that obstructed the filtration angle over 
an area from one-fourth to one-third of its circumference. Figure 10 shows a case in which stripping successfully normalized pressure. Analysis of this series of cases suggests that stripping of this amount of the angle is sufficient and is the sine qua non of successful normalization of pressure. It also suggests that stripping of only a part of the circumference is adequate to permit the pressure regulating mechanism to assert itself and permanently to maintain normal tension and function. Since this effect has been observed in individual cases as long as 10 years after goniotomy, and since cases of recurrence of increased pressure have been the exception, it appears that the effect is permanent.

In all bilateral cases in this series and in most monocular cases, judging from results obtained by stripping the angle and by preoperative and post-operative gonioscopic examination, the initial increased pressure appeared to be the result of obstruction of the angle due to arrested regression of the uveal meshwork. Since removal of the persistent embryonic tissue resulted in normalization of pressure, it must be assumed that Schlemm's canal was present. There was no sign that either internal cyclodialysis or external filtration had taken place in any of these cases. One may conclude, therefore, that the mode of action of goniotomy consists in restoring access of aqueous to Schlemm's canal by removing obstructing tissue.

In one case there was direct gonioscopic evidence of the presence of Schlemm's canal in that portion of the angle from which the obstructing tissue had been removed. This consisted of a pink band which was seen to lie external to the trabeculum in the position of Schlemm's canal. In the adult this pink band has been shown by Kronfeld to be produced by blood in Schlemm's canal ${ }^{4}$. The presence of Schlemm's canal is suggested by the post-operative gonioscopic findings in the aforementioned successful cases. It is confirmed by the analysis of anatomical examination of 84 specimens taken from unoperated eyes, as reported by Anderson ${ }^{5}$. He found that Schlemm's canal was present in 75 per cent. of the earliest specimens. No sign of it was found in more than half the specimens taken from children over $2 \frac{1}{2}$ years of age. He suggests that the canal becomes closed in the later stages as the result of distension of the eyeball and of increased intra-ocular pressure. The evidence obtained from these specimens thus confirms the rationale based on gonioscopic examination of the effectiveness of stripping the angle in early congenital glaucoma.

\section{Urgent NeEd For Early Operation}

The mode of action of the operation as explained in the foregoing shows the importance of early diagnosis for a successful 
outcome of operation, and explains why the chances of establishing outflow with this operation may be expected to diminish with the duration of the condition. It is essential to operate early before prolonged distension of the eyeball has caused obliteration of Schlemm's canal. Other important reasons for early diagnosis and prompt operation are :

1. Restoration of vision by means of clearing the cornea. Corneal cloudiness is largely reversible in the early stages, progressively less so in later stages.

2. Prevention of amblyopia due to prolonged obstruction of vision by cloudiness of the cornea.

3. Prevention of permanent scar formation developing from corneal cloudiness.

.4. Prevention of injury to the optic nerve caused by prolonged pressure.

In general, it is important to prevent progressive enlargement of the eyeball before distension has produced permanent changes and to encourage development of normal anatomy and of physiological function during the period of growth.

Results

The results of goniotomy* in congenital glaucoma are summarized in the following tabulation :-

TABLE I

\section{Goniotomy in Congenital Glaucoma}

$\begin{array}{lllllll}\text { Number of infants and children } & \ldots & \ldots & \ldots & \ldots & 5 \text { I }\end{array}$

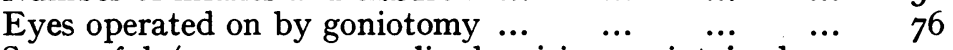

Successful (pressure normalized; vision maintained or $\begin{array}{llllllll}\text { restored) } & \ldots & \ldots & \ldots & \ldots & \ldots & \ldots & 66\end{array}$

$\begin{array}{llllllll}\text { Unsuccessful } & \ldots & \ldots & \ldots & \ldots & \ldots & \ldots & \text { 10 }\end{array}$

Eyes on which goniotomy was not applicable $\quad \ldots \quad$...

It has been my custom to prescribe miotics three times daily for two months after surgery. The result of the operation was considered successful when intra-ocular pressure never rose higher

* Goniotomy in infants and in adults is two distinct operations which differ in rationale, technique and effectivity, In infants the operation, which has been developed to a relatively satisfactory conclusion, consists of removing occluding foetal meshwork from the angle. In adults, in whom it has not yet shown an adequate degree of consistent efficacy to recommend its employment except in the occasional case, its objective is to incise the angle wall, that is the trabeculum proper. For this reason it is suggested that the term goniotomy be applied only to the operation performed on infants, and goniotrabeculotomy or trabeculotomy to the procedure carried out on adults. 
than $35 \mathrm{~mm}$. of mercury with the McLean or $21 \mathrm{~mm}$. with the Schiötz tonometer three months after operation, no miotics having been used during the two weeks preceding measurement of the pressure.

Among the successful cases the time elapsed since the date of operation varied from six months to ten years. There were recurrences in two eyes, mentioned in the next paragraph. The oldest case (two eyes) was operated on ten years ago; 5 eyes seven years ago; 8 eyes six years ago; 21 eyes from four to six years ago; 11 eyes from two to four years ago; 9 eyes from one to two years ago and 10 eyes from three to twelve months ago.

The recurrence of increased pressure which took place in two eyes was relieved by a second goniotomy in both instances. In F.W. pressure increased in the left eye eight years after operation. In J. McL., pressure increased in the left eye four years after operation. Gonioscopic examination showed that the stripping of the angle had been insufficient.

Repeated operations were necessary in 22 eyes before pressure was permanently normalized. One operation sufficed in each of 45 eyes. In some cases, for example that of P.O'Q. (Fig. 1), goniotomy was performed four times on each eye before permanent normalization was attained. The ultimate result was excellent. I believe it is better to err on the conservative side and partially strip the angle with safety on repeated occasions than to attempt to do an extensive stripping at one operation at the cost of greater hazard. Cases which required repeated operations before permanent normalization was attained are not listed as recurrences.

The operation was performed under the contact glass on 20 eyes. All of these were successful. Repeated operation is the exception when operating under the contact glass. In 56 eyes the operation was performed without the contact glass because of corneal cloudiness. On 18 of these repeated operation was necessary.

In 8 eyes which had been ineffectively trephined goniotomy normalized tension.

\section{Advantages, Disadvantages and Hazards}

Striking among the results of goniotomy are absence of cosmetic disfigurement and preservation of a round central freely reacting pupil.

A disadvantageous feature of the operation without the glass is the need to repeat the operation in one-third of the cases in order to obtain permanent normalization of tension.

Hazards of the operation are excessive haemorrhage or iridodialysis if the root of the iris is incised. Vision was lost in two 
eyes early in this series as the result of excessive haemorrhage. Recently one eye in a congestive neglected case of a $2 \frac{1}{2}$ year old child was lost as the result of a massive intra-ocular haemorrhage which occurred on the sixth post-operative day. Infection, as in any other intra-ocular operation, is a possible complication but was not encountered in this series. A severe reaction occurred in one case which could be considered an infective iritis. As always the eyes had been operated on different days, in this instance after an interval of three weeks. The condition cleared after 18 days leaving in one eye a small central opacity of the anterior capsule; in the other an anterior adhesion produced eccentricity of the pupil. Tension was normalized and it is evident that useful vision is developing. Sympathetic ophthalmia has occurred in no case up to the present time.

\section{INDICATIONS}

Goniotomy is indicated in all bilateral and unilateral cases of congenital glaucoma in which increased pressure is the result of obstruction of the angle by persistent embryonic tissue, provided that Schlemm's canal has not been obliterated by prolonged distension of the bulbus. This includes the majority of cases of congenital glaucoma in the early stages. Generally speaking, the earlier it is applied after onset of symptoms the more effective is the operation in maintaining and restoring vision. Results may be dramatic when the bulbus is in the early congestive phase associated with cloudiness of the cornea. In these cases the urgent need for early diagnosis and prompt adequate operation can hardly be over-emphasized. There are cases, however, which run a chronic course during which the corneae remain clear, the optic nerve undergoes little damage and the bulbus is only moderately enlarged after a long period of time. Good central vision is maintained in them. Since the corneae are clear goniotomy can be performed under direct vision with the aid of the glass, and may be effective several years after the onset of symptoms. Excellent results were obtained in some of these cases at 4 and 6 years of age with corneal diameters of $14 \mathrm{~mm}$.

\section{CONTRA-INDICATIONS}

Goniotomy is contra-indicated in older children with enlarged eyes, that is, in eyes in the advanced stage which have had a congestive history with consequent corneal scarring and cloudiness and a corneal diameter of $15 \mathrm{~mm}$. or more. In these enlarged "buphthalmic" bulbi the danger of haemorrhage is greatly 
increased because of dilated vessels and collateral circulation which has become established. Haemorrhage is absorbed more slowly, no doubt due to degenerative condition of the eyes and to their changed circulation. Schlemm's canal may be obliterated as a result of the distension.

When permanent obliteration of the canal'has taken place and when repeated goniotomies have proved ineffective, iridencleisis or cyclodiathermy may be tried.

Some rare unilateral cases of congenital glaucoma appear to be the result of other kinds of anomalies, such as an aberrant reflection of the iris stroma to an insertion at the anterior border ring. The extremely rare cases of narrow angle (angle or iris-block) glaucoma ${ }^{6,7}$ in small children do not come within the scope of this article since goniotomy is not applicable to them.

\section{TeChNique}

There are two major procedures of goniotomy: if the cornea is clear goniotomy is performed by direct vision under a prismatic contact glass; if the cornea is cloudy it is done without the glass. The general considerations which the procedures have in common will be treated first.

\section{General Considerations}

Pre-operative measures. Paediatric examination including X-ray of the chest for enlarged thymus is performed in all cases. In the case of infants, the usual diet is maintained up to 6 hours preoperatively; sugar solution is forced up to 4 hours pre-operatively to avoid dehydration with associated hyperpyrexia. In order that surgery may not be delayed and since ether may have to be given several times in the course of the following weeks, every effort should be made to prevent the infant from catching cold. Miotics, preferably prostigmine 5 per cent., are continued usually every 3 hours while the patient is awake until $1 \frac{1}{2}$ hours before the time of operation. One extra drop is instilled three times at intervals of half hours before operation in the eye to be operated upon. If circumstances permit one drop of sulmefrin is instilled t.i.d. for several days pre-operatively.

Anaesthesia. In view of the delicacy of the operation as applied to infants, which may be only a few days or weeks old, anaesthesia is an important factor. A preliminary injection of atropine is given 45 minutes pre-operatively. Ether is administered through a small airway (intrapharyngeal insufflation). A generous supply of oxygen should be assured at all times. While the patient is being 
prepared the lids must be kept closed with moist pledgets but without pressure, since the cornea in congenital glaucoma is extremely susceptible to exposure and to pressure. For the same reason the heat generated by operating room lamps should be avoided. Anaesthesia must be sufficient at the moment of operation in order that there be no movement of the patient. In children over six months of age a small dose of paraldehyde is administered rectally 45 minutes pre-operatively. Post-operative agitation which may follow ether anaesthesia is not harmful since there is only a small obliquely placed puncture wound which does not permit egress of ocular contents. Before the patient is draped pressure is taken with a tonometer, the base of which has been sterilized. The corneal diameters are measured, and the condition of the cornea and size of the pupils noted. Sometimes the decision of which eye to operate upon can be made only at this time.* Then the position of the patient, operator, assistant, anaesthetist and instrument table is arranged accordingly.

Position of Patient. The infant is placed on a circumcision board : for the right eye on the centre of the operating table; for the left eye with the head end of the board protruding obliquely off the table. For operation under the glass the eye should be 47 inches above the floor to permit a convenient position for an operator 5 feet 10 inches in height. For goniotomy without the glass the patient's eye should be 46 inches or less above the floor in order that the operator may look vertically down upon it. A pillow or towels are placed under the head bringing it into a horizontal plane. The anaesthetist maintains the head in position during the operation.

Position of Operator and his Assistants. The operator stands on the side of the eye to be operated on : for the right eye at 11 o'clock ; for the left eye, at 4 o'clock. The illuminator holding a hammer lamp must be high enough, standing on a platform if necessary, to obtain a good view of the field of operation. He stands on the same side and to the right of the surgeon for the operation with the glass, on the opposite side for operation without the glass. The assistant fixates the bulbus with two Gifford forceps with lock, standing at 3 o'clock for the right eye and behind the head for the left eye. The anaesthetist is on the side opposite the operator, for the right eye at 5 o'clock in order not to interfere with the assistant who is standing at 3 o'clock.

Magnification. The operator uses a +2.50 sphere hanger over his correction. He wears a binocular head loupe +5.00 sphere

\footnotetext{
* In cases in which the decision has already been made the taking of the tension is dispensed with as a preliminary procedure in order to reduce the time of anaesthesia.
} 
(Hess head loupe) which he uses only in case the anterior chamber is deepened through a corneal puncture made with a discission knife. He does not use it for the goniotomy.

Illumination. The room is in semi-darkness. One lamp situated at some distance from the table and directed away from it is in readiness for general utility purposes. The field of operation is

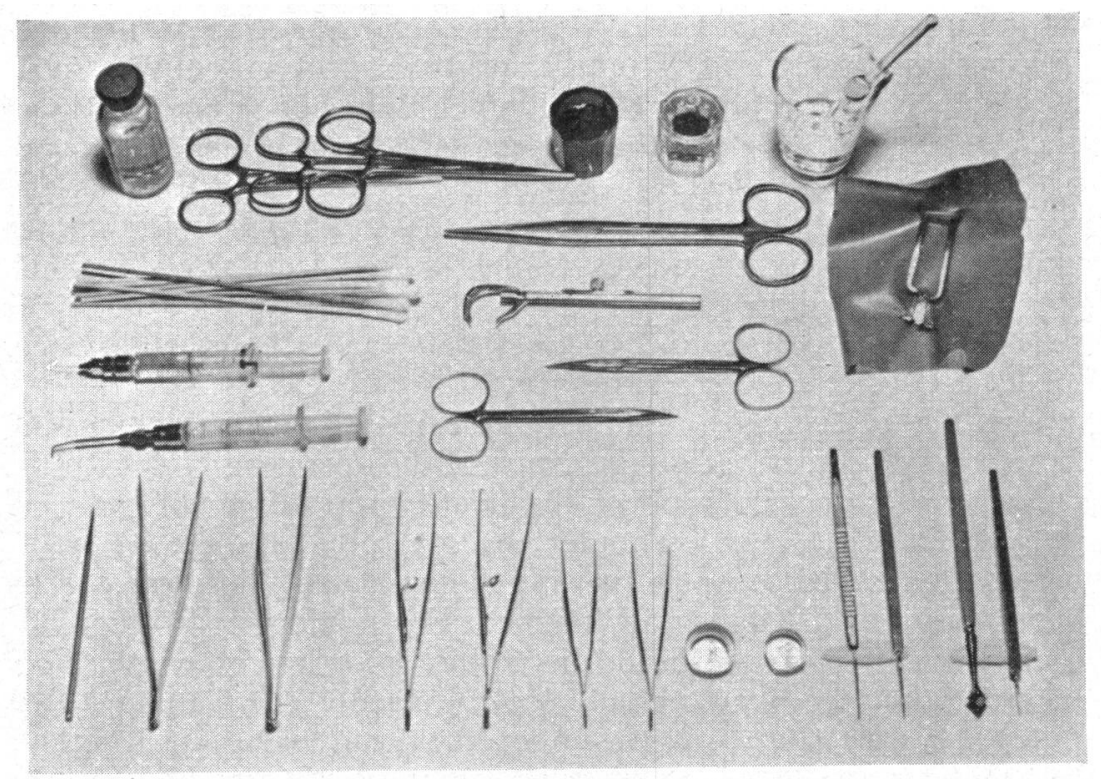

FIG. 11

Usual set-up tray for Goniotomy. Ampoule of physiological saline. Haemostats, mosquito. Dappen glass containing tincture of iodine. Dappen glass containing solution fluorescein, 1 per cent. Medicine glass containing physiological saline and glass rod. Speculum with rubber dam. Applicators. Mayo scissors. Luer-Lok syringe, 2 c.c. with * 30 needle, containing physiological saline solution.-Caliper. Stevens' scissors. Lacrimal dilator. Splinter forceps. Gifford's forceps with lock. Bishop-Harman's forceps. Surgical contact glass (small and large). Goniotomy knives. Keratome. Discission knife, narrow.

illuminated by a hammer lamp* which is made of light plastic and is air cooled. It is used in both procedures but manipulation differs respectively. The illuminator must be familiar with and trained in the procedure. The technique of illumination is especially important in goniotomy by direct vision under the glass and must be carefully rehearsed before the operation.

Instrument tables. Small and large instrument tables are on the side of the eye to be operated upon (Fig. 11).

* This lamp was demonstrated at the meeting of the A.M.A. in San Francisco, July 4, 1946 A description of it is in publication. 
Preparation of Patient. Lashes are clipped (with Stevens' scissors and ointment, wiped off with cotton spindle after each clip). The face is prepared and the eye irrigated in the usual manner. After the patient is draped a face mask, which should be of moistened gauze in order to be easily moulded to the region, is applied.

\section{Goniotomy Under The Contact Glass}

Once the glass has been applied, time is the essence of goniotomy. A complete check, therefore, is made beforehand in order to assure that everything is in readiness and in position on the instrument table. The speculum is passed through two holes made in a piece of rubber dam 6 inches square. The height of the assistant and of the illuminator is checked. Either or both may require a platform to stand on. The lids are kept closed throughout the preparation. A few minims of adrenalin 1:1000 are injected with $a=1=30$ needle and 1.5 c.c. Luer syringe into the outer canthus; after one minute a canthotomy is performed with straight Mayo scissors. The quartz surgical contact glass which has been cold sterilized is dried and placed on gauze in a small bowl on the instrument table. The knives ( 2 goniotomy and 2 discission knives) are now placed on the table, points toward the operator.

The operator stands at 11 o'clock for the right eye and at 4 o'clock for the left eye. The platform upon which the illuminator is about to stand is already in position to the operator's right. The instrument table and surgical nurse are also to the right of the operator. The speculum is now inserted and the rubber dam trimmed especially on the nasal side. On the temporal side the dam covers the lids, preventing contact of the knife with them and relieving the operator's mind of this important detail. The cornea is moistened when necessary with physiological saline solution applied by means of a glass rod. The eye is fixated by the assistant 2 to $3 \mathrm{~mm}$. posterior to the corneo-scleral border at 12 and 6 o'clock by means of two Gifford forceps with spring lock. He must be mindful not to touch the lock lest it spring open during the operation. The head and eye are rotated away from the surgeon who applies the contact glass in the usual manner by injecting physiological saline solution between it and cornea through a Becton, Dickinson curved gold canula and 1.5 c.c. Luer syringe.

When operating on the right eye (Fig. 12) the fixator's right hand holds the forceps at 12 o'clock, the left at 6 . The left hand must be in such a position that it does not obstruct the view of the illuminator as he moves counter-clockwise. When operating on the left eye, the right hand is at 6 o'clock and the left at 12 . 


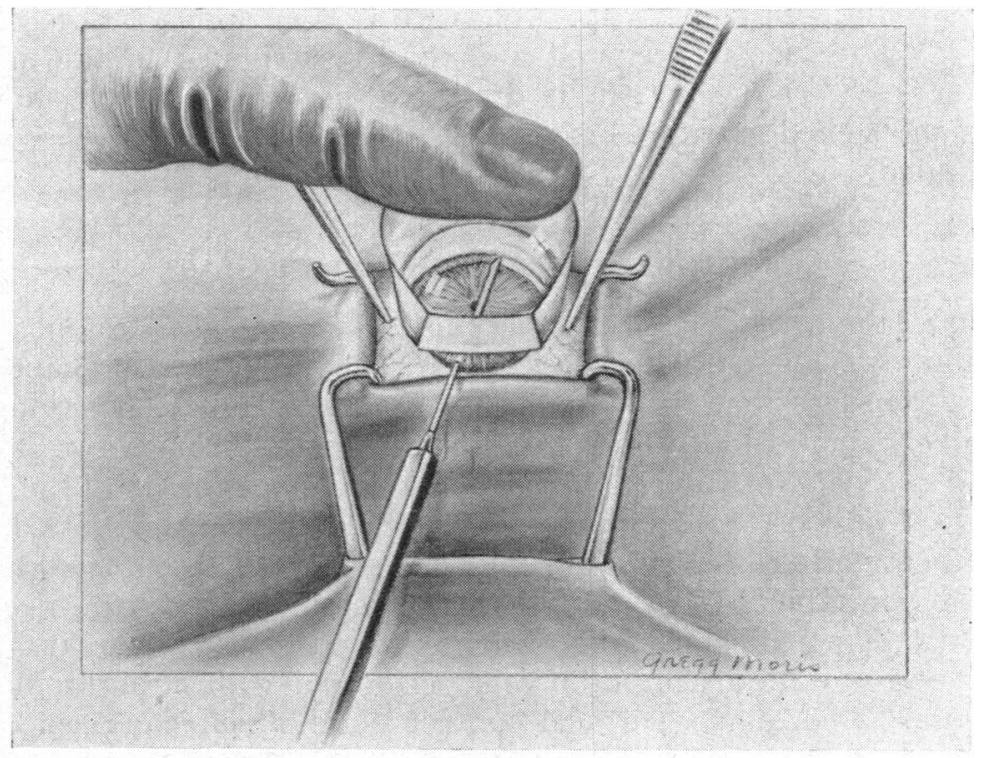

FIG. 12

Drawing shows goniotomy or stripping of the angle under the glass. The root of the iris retracts behind the blade, leaving a white wake behind it which is the wall of the angle.

Regardless of the side under operation, the fixator, unless tall, should stand on a platform in order to assure visibility of the eye throughout the procedure and to prevent the tendency arising from poor visibility to retract or dimple with the forceps. The surgeon supports the glass with the index finger of his left hand. Two indentations on top of the glass prevent the finger from slipping. The fixator abducts the eye slightly in order to expose a strip of the temporal portion of the cornea 2 or $3 \mathrm{~mm}$. wide where the puncture is about to be made. By abduction the eye can be made to offer resistance to the glass when this is pressed more firmly against it by the operator's finger at the moment of puncture, thus preventing ingress of air at this critical moment. The operator applies the end of a cotton applicator previously dipped in tincture of iodine at the intended site of puncture. The goniotomy knife is then passed to him by the instrument nurse. She retracts the instrument table and the illuminator assumes his position on the platform immediately to the operator's right.

The illuminator stands on the platform sufficiently far removed from the surgeon to provide room for him to sway counter- 
clockwise during the progress of the incision. The illuminator must stand with feet well apart in order to be able to sway in unison with the counter-clockwise movement of the surgeon without having to take a step. He maintains the lamp in contact with the temple of the surgeon, at the same time looking down the top of the shaft of the lamp, so that he may have the same view of the angle and of the blade of the knife as the surgeon. The heads should maintain contact. Perfect synchronization of heads and light is necessary; they must move as one unit. A light on a head band did not give as good results as the technique described.

The eye first having been rotated 2 hours counter-clockwise puncture is made in the right eye at 10 o'clock, in the left at 4 o'clock, $1 \mathrm{~mm}$. anterior or axial to the corneo-scleral border and oblique so that the corneal wound is valve or trapdoor-like. At the moment of puncture, extra pressure is exerted by the operator's index finger on the glass in order to prevent ingress of air. Pressure must be exerted in the direction of the optic axis as tilting of the glass permits entrance of air. For the same reason dimpling of the sclera with the fixator's forceps must be avoided. The operator guides the knife across the pupil to the opposite side. In traversing the chamber the knife must always cross the optic axis. When the knife crosses the optic axis the diameter of the arc described after the opposite angle is engaged will be greater than the diameter of the circumference of the limbus. Therefore, the point of the knife remains engaged. When the knife does not cross the optic axis, the contrary holds true. In describing a smaller arc than that of. the limbus, the point of the knife becomes disengaged and too small an incision is made. Moreover, the extra movement of advancing the knife necessary to maintain contact with the angle wall is prone to result in picking up iris tissue near the root with consequent iridodialysis and haemorrhage. The blade is inserted just anterior to the root of the iris and is moved counter-clockwise in this plane as long as visibility permits. This usually amounts to several millimetres or from one-fourth to onethird the circumference. During this excursion of the blade, the shaft is rotated around its axis in a clockwise direction to encourage a stripping action and to prevent the blade from moving posteriorly out of the intended plane into the dangerous region of the ciliary body. During stripping under the glass, the root of the iris is seen to retract behind the blade, leaving a white wake behind it which is the wall of the angle. In some cases this has the appearance of a cut piece of parchment paper that drops backward as the incision proceeds. Stripping should be under complete control and placed immediately above the root of the iris, care being exercised to avoid blood vessels at the iris root which in some cases 
are clearly visible. There may be a slight sensation of grating as the blade proceeds; the fixator may recognize the need of exerting slight resistance. Since picking up the root of the iris causes haemorrhage it is advisable to start well anterior to the root and to strip or peel off the insertion of the iris from above. When stripping has been completed the knife is quickly removed, without loss of aqueous in most cases, care being taken to avoid enlarging the puncture wound in the cornea by slight pressure against the back of the blade during its removal. The contact glass and fixating forceps are withdrawn. After a few seconds a slight oozing of blood of venous colour appears in the anterior chamber along the line of stripping; this is absorbed within a few hours. If after removal of the knife the pupil should be eccentric, the cornea is tapped near the puncture with a spatula or the tip of a lacrimal dilator is inserted to prevent adhesion of iris to the inner wound lip.

The speculum is removed and the canthotomy closed with catgut. Eserine ointment 1 per cent, and sulphathiazole ointment 10 per cent. are inserted into the conjunctival sac. Binocular pads are applied, that over the operated eye being covered by a metal shield. The child is placed in bed on the operated side in order that any blood may settle on the opposite side of the chamber. Arm cuffs are applied. In older children, restrainers may be necessary. Elixir phenobarbital dram 1 may be indicated. After 24 hours it has been customary in bilateral glaucoma to instil prostigmine 5 per cent. in the unoperated eye and after 48 hours in the operated eye; thereafter drops are continued three times a day in both eyes. Only one eye is operated on at a time.

If the different steps of the procedure have been reviewed preoperatively there should be no loss of time at operation so that once the glass is applied goniotomy is quickly completed.

If goniotomy under the glass is not feasible, the operation is performed without the glass. Operating under the contact glass is desirable whenever possible. In the past various procedures (use of glycerin, hypertonic salt solution, retrobulbar injection of adrenalin $1: 1000$ inter al.) have been tried without success with a view of clearing a cornea which has become cloudy during pre-operative manoeuvres, application of forceps and glass. Letting off a small amount of aqueous through a valve-like corneal puncture made with a Graefe knife was also tried. It cleared the cornea but did not permit of adequate operation under the glass because of complicating circumstances. Recently a preliminary operative abrasion of the epithelium prior to application of the contact glass has enabled oberation under the glass in cases in which it has heretofore been impossible. 


\section{Goniotomy Without the Contact Glass}

The preparatory measures already outlined for goniotomy under the glass are carried out. The precautions mentioned under general consideration are observed. The height of the eye should be such that the operator looks vertically down upon it. Illumination is provided by the hammer lamp carried on the end of a rod so that the light falls as nearly vertically from above as possible. The illuminator stands opposite the surgeon, for the right eye at 4 o'clock and for the left eye at 10 o'clock. The rubber dam on the nasal side must be trimmed well back to avoid casting a shadow on the field of operation. The bulbus is fixated with two forceps held by the assistant, in the same manner as for the operation under the glass. The forceps should be vertical to the sclera and must include episcleral fibres in order to permit of resistance or counter-pressure on the part of the fixator during stripping. Additional fixation by the operator with a Bishop Harman forceps held in his left hand is helpful. If the assistant is not sufficiently experienced with this operation, it is best to divide the fixation between him and the surgeon. In this case the surgeon fixates with the Gifford forceps with lock in his left hand at 12 o'clock on the right eye and at 6 o'clock on the left, while the assistant fixates with his left hand at 6 o'clock on the right eye and at 12 o'clock on the left.

Canthotomy is indicated in most cases. In small infants, on whom the operation is especially delicate, the head is rotated toward the surgeon and the eye slightly abducted in order to bring the nasal limbus into a frontal plane to the surgeon's line of regard. In the case of a greatly enlarged eye this lateral movement is limited because the temporal limbus disappears as the result of even slight abduction. The surgeon applies Bishop Harman forceps at the contra-lateral limbus, helping to fixate and guide the rotation from there.

The puncture in the cornea is made as already described, being sure that it is oblique (valve-like) in order to encourage retention and reformation of the anterior chamber. It is made on the right eye at 10 o'clock, the eye having first been rotated counter-clockwise 2 hours. The blade of the knife crosses the pupil and disappears behind the limbus on the opposite side in a plane just anterior to that of the iris. As it engages the angle wall and starts its excursion in a counter-clockwise direction slight resistance is felt. Its tip can be seen through the sclera from the outside. Seen through the cornea, the knife appears $0.5 \mathrm{~mm}$. farther anterior than it is in actuality. This appearance must not influence the surgeon to guide his knife farther posteriorly. A posterior position 
of the blade is the greatest hazard of the operation. It is better to err on the conservative side by maintaining a more anterior plane at the risk of not stripping the angle. The operation can always be repeated. Rotation of the knife clockwise around its own axis helps to prevent it from being guided or from slipping to a posterior position. Stripping the angle wall is usually associated with a feeling of slight resistance and of grating if the blade is not too sharp. The absence of this feeling is an indication that the blade is too far posterior and must be avoided. If indicated the knife may be reversed and the stripping repeated in the opposite direction.

If upon re-examination later this goniotomy proves to have been insufficient, another can be performed on an adjoining part of the angle after suitable rotation of the eyeball. It has been possible in some cases, as shown by post-operative gonioscopy, to strip the angle over almost one-half its circumference at one sitting. Picking up the root of the iris and haemorrhage are the only serious complications to be guarded against. It has been found that removal of blood from the anterior chamber by paracentesis and irrigation cannot be adequately accomplished in infants. It is better to be obliged to repeat the operation than to provoke a major haemorrhage by too posterior position of the blade or by attempting too extensive stripping. If the stripping has been properly placed a little blood of venous colour begins to ooze from several points along the line of stripping a few seconds after removal of the knife. If the head is rotated to the opposite side, the blood coalesces to a thin sheet in the region of the pupil and is seen to stop. If the blood is more arterial in colour, is more extensive and follows immediately upon the incision, it is an indication that the latter has been placed more posterior than is desirable. Blood may fill one-half the chamber; it is usually absorbed within 24 to 48 hours.

The canthotomy wound is sutured, medications given and dressing applied as already outlined in the procedure under the glass.

Notes on Stripping of the Angle. In congenital glaucoma, there is an area of from 2 to 3 hours in the circumference of the angle opposite the point of puncture in which it is easiest to make an adequate stripping. Since the position of the puncture is limited on the right eye by the brow and on the left by the cheek bone, a point is chosen as far as possible in a clockwise direction-for instance, on the right eye at $\mathbf{1 0 . 3 0}$ o'clock in order to strike the opposite nasal angle as far clockwise as possible, i.e., 4.30 o'clock. When the blade has reached 2.30 o'clock it has covered the area in which stripping can be done under optimum conditions and with least hazard. Post-operative gonioscopy shows that it is often possible 
to strip a more extensive area, up to 4 hours or more by the clock. Since this cannot be done consistently and since it is associated with greater hazard of haemorrhage, it is well to consider allocating the area of the first stripping in such a way that another area of the angle as yet untouched can be stripped on a second occasion. For this purpose it is advisable in both procedures, with and without the glass, to start with the eye rotated 2 hours in a counterclockwise direction. Thus for operation on the right eye the bulbus is rotated in a counter-clockwise direction so as to bring the area between 6 and 3 of the nasal angle within operative reach. The assistant maintains the bulbus in this position and resists, if necessary, the traction of the blade while it strips the angle in a counter-clockwise direction. If later examination shows that the result of this procedure was not sufficient to normalize pressure, stripping of a neighbouring area of the angle (from 3 to 12) may be undertaken. In the latter case the right eye is rotated two hours in a clockwise direction to make the area accessible. The puncture is made at $10 \cdot 30$ o'clock and stripping is performed from 5 to 3 o'clock in the rotated position which corresponds to 3 to 1 o'clock when the bulbus is in the normal position. It is possible, therefore, by means of two goniotomies to strip the angle up to one-half its circumference, i.e., from the 6 to 12 meridians.

Preliminary Deepening of the Anterior Chamber with Physiological Saline Solution. Preliminary deepening of the anterior chamber with physiological saline solution may be performed in cases operated on without the glass in order to minimize the hazard of picking up the root of the iris with the tip of the knife. The chamber is not deepened for the operation under the glass; in this case the blade of the knife is guided under direct vision and therefore picking up of the iris root with consequent haemorrhage can be avoided. Deepening should be considered in infants, especially those between the ages of several days to weeks because of the relatively shallow chamber and narrower angle. It permits of a more deliberate placement of the stripping in regard to its anteroposterior position. However, it is well to dispense with deepening when possible as it introduces a delicate technical detail which, if not adequately performed, may result in leakage of aqueous and postponement of the operation. When deepening is employed great care must be taken in prelaying the corneal puncture. The bulbus is fixed with a Bishop Harman forceps at the opposite limbus. The cornea is punctured very tangentially $1 \mathrm{~mm}$. axial to the corneo-scleral border at 9 o'clock on the right eye and at 3 o'clock on the left eye, with a discission knife, the blade of which has been previously dipped in fluorescein. The wound canal should 
be at least $3 \mathrm{~mm}$. long. The tip of the knife should barely perforate Descemet's membrane as observed through a five times magnifying head loupe. It may be necessary to tip the knife backward a little in order to perforate this membrane. Care must be taken not to enlarge the puncture upon removing the discission knife lest leakage ensue. The puncture made visible by fluorescein is touched with a dry applicator and one dipped in tincture of iodine from which the excess has been removed. With a = 1 30 needle (previously drum tested or examined under a loupe to assure its having a perfect point) attached to a 1.5 c.c. Luer syringe intravenous saline solution is injected, the surgeon fixing the bulbus at the contra-lateral limbus with a Bishop Harman forceps.

\section{Air Goniotomy}

If goniotomies have not reduced the pressure sufficiently and gonioscopy shows that further repetition of goniotomy in the nasal portion of the angle is not feasible because of iris adhesions or scar formation, an attempt may be made to perform a goniotomy under air in the temporal portion of the angle. This area can be made visible by filling the chamber with air. ${ }^{8,9}$ Since it is difficult consistently to insufflate the whole anterior chamber with air through a corneal puncture, an oblique scleral incision as for cyclodialysis is used and the sterile air injected by means of a curved gold cannula and small Luer syringe. The eye is then fixated with two Gifford forceps with lock and abducted. 'The temporal limbus disappears behind the canthus. The operator stands on the opposite side. The knife is passed across the bridge of the infant's nose and the cornea punctured $1 \mathrm{~mm}$. axial to the nasal corneo-scleral border. Goniotomy under air or " air goniotomy " of the temporal portion of the angle is performed. Only a gross picture of the insertion of the root of the iris is discernible through air, due to the opacities of the cornea, to reflections and to absence of magnification. However, visibility may be sufficient when combined with familiarity of the angle to act as a guide to the blade of the knife.

Blunt Dissection. Blunt dissection of the angle with a spatula inserted through a prelaid corneal puncture has been tried in place of stripping or peeling of the angle with a goniotomy knife. In practice this has shown no advantage over the present technique with the knife; on the contrary it appears to encourage adhesion of the root of the iris and closure of the angle.

* The knife, surgical contact glass and hammer lamp can be obtained from A. H. Parsons Laboratories, 442 Post Street, San Francisco, 2, California. The knife will be obtainable also from E. Grieshaber, Schaffhausen, Switzerland. Recently specifications for its present form have been sent to V. Mueller and to Storz. 
Goniotomy Knife. The shaft of the knife is conoid in order to prevent loss of aqueous. Its diameter increases progressively from the tip of the blade to the heel of the shaft. The blade is not too sharp as it is used to strip or peel and not to incise, the tip being sharp enough, however, to permit easy puncture of the cornea. Some resistance is felt during the stripping, which gives the operator a feeling of guidance and deliberate movement.

\section{SUMmary}

Congenital glaucoma in the past has resulted in a high percentage of blindness, due to lack of an adequate operation.

Goniotomy, performed at this writing on 76 eyes afflicted with infantile glaucoma, preserved useful vision in most cases. In 66 eyes pressure was normalized and vision maintained or restored over periods ranging from one to ten years. In ten the operation was unsuccessful. When combined with early diagnosis it provided excellent visual results.

A plea is made for early diagnosis and prompt, adequate operation by goniotomy.

The symptoms of increased intra-ocular pressure in congenital glaucoma may be present at birth, or their onset may be rapid or sudden during the first few months of infancy. In most cases symptoms consist of cloudy cornea, photophobia and other signs of irritation and congestion. Diagnosis should be made as soon as possible after onset of congestive symptoms and not deferred until enlargement of the eye is evident. In the rare cases of slow onset there are no congestive symptoms. Pressure should be measured with a tonometer under ether anaesthesia, which must be sufficient to assure complete relaxation at the moment of measurement.

The appearance of congestive symptoms is prone to lead to a faulty diagnosis of blepharitis, conjunctivitis or keratitis, causing loss of valuable time and bearing tragic consequences.

The initial cloudiness of the cornea is associated with roughening of the corneal epithelium. This disturbance of the cornea is the chief cause of the irritative symptoms and photophobia. If the corneal cloudiness is allowed to persist, it is superseded by permanent scarring with associated irregular astigmatism and ensuing amblyopia of greater or less degree.

Glaucomatous atrophy of the optic nerve was the exception in this series of infants and children. It occurred only in those few cases in which normalization of pressure was unduly delayed. In infants it is not nearly as urgent to protect the optic nerve-head from pressure as it is to relieve cloudiness of the cornea. 
If soon after its inception increased intra-ocular pressure is normalized by goniotomy, transparency of the cornea is almost completely restored and maintained, and vision is afforded the opportunity to develop. When this is borne in mind, the urgency of prompt relief of increased pressure is evident.

The mode of action, advantages and disadvantages, indications and contra-indications of goniotomy are given.

The technique of goniotomy is described.

The author wishes to express his appreciation to his colleagues who have referred these patients, as well as to Childrens' Hospital, San Francisco, for its helpful co-operation,

\section{REFERENCES}

1. Barkan, Otro.-Operation for congenital glaucoma, Amer. Jl. Ophthal., Vol. XXV, pp. 552-568, May 1942.

2. Goniotomy for congenital glaucoma, Amer. Jl. Ophthal., Vol. CXXXII, pp. 526-533, February, 1947.

3. Anderson, J. R.-Hydrophthalmia or congenital glaucoma, Cambridge University Press, p. 104, 1939.

4. KRonfeld, Peter C. et al.-Gonioscopic studies on the canal of Schlemm, Amer. Jl. Ophthal., Vol. XXV, pp. 1163-1170, October, 1942.

5. Anderson, J. R.-Hydrophthalmia or congenital glaucoma, Cambridge University Press, p. 151, 1939.

6. Barkan, Otto,-Glaucoma, classification, causes, and surgical control, Amer. Jl. Ophthal., Vol. XXI, pp. 1099-1117, October, 1938.

7. —_ An operative procedure for glaucoma of shallow chamber type, Arch. Ophthal., Vol. XXI, pp. 331-345, February, 1939.

8. - Goniotomy, Amer. Jl. Ophthal., Vol. XXVIII, pp. 1133-1134, October, 1945.

9. Hughes, Wendell L., et al.-Technical uses of air in ophthalmology, Arch. Ophthal., Vol. XXXV, pp. 525-537, May, 1946. 